\title{
An efficient synthesis of $\mathbf{2 H}$-1,4-benzoxazin-3(4H)-ones via Smiles rearrangement
}

\author{
Jongphil Kang, ${ }^{\text {a Kyeong-Hee Kam, }}$ Manjunath Ghate, ${ }^{a}$ Zuo Hua, ${ }^{a}$ Tae-Hyun Kim, \\ Ch. Raji Reddy, ${ }^{c}$ S. Chandrasekhar, ${ }^{c} *$ and Dong-Soo Shin ${ }^{\mathrm{a} *}$ \\ ${ }^{a}$ Department of Chemistry, Changwon National University, Changwon, GN, 641-773, Korea \\ ${ }^{b}$ Department of Chemistry, University of Incheon, Incheon, 402-749, S. Korea \\ ${ }^{c}$ Organic Division-I, Indian Institute of Chemical Technology, Hyderabad-500007, India \\ E-mail:1sshin@changwon.ac.kr,srivaric@iict.res.in
}

\begin{abstract}
A novel and effective synthesis of substituted 1,4-benzoxazinones via Smiles rearrangement is described. Treatment of $N$-substituted 2-chloroacetamide, substituted 2-chlorophenols and cesium carbonate in refluxing DMF afforded the corresponding substituted 1,4-benzoxazinones in excellent yield.
\end{abstract}

Keywords: 1,4-Oxazinone, Smiles rearrangement, 1,4-benzoxazin-3-one, cesium carbonate

\section{Introduction}

1,4-Oxazinones are very important structural motif, which has wide application in medicinal chemistry due to its pharmacological properties. ${ }^{\text {la-c }}$ The members of this family are used for treating Parkinson's disease, ${ }^{1 \mathrm{~d}-\mathrm{e}}$ ischemia reperfusion, ${ }^{2}$ selective potassium channel openers, ${ }^{3}$ antinociceptive, ${ }^{4}$ antidepressant ${ }^{5}$ and antifungal agent. ${ }^{6}$ In addition the fluorescent properties of 1,4-oxazinones are valuable as laser dyes $^{7}$ and coupling agent for oxidative hair dyes. ${ }^{8}$ Moreover, 1,4-oxazinones used as intermediates for the synthesis of aza sugars ${ }^{9}$ and ambident cycloaddition reactions of these compounds were well established. ${ }^{10}$ Despite their importance from pharmacological, industrial, and synthetic point of view, comparatively few methods for their preparation were reported. Some of the methods reported in the literature for the synthesis of oxazine derivatives are McKillop cycloaddition, ${ }^{11} \mathrm{~N}$ - and O-alkylation with dibromo compounds, ${ }^{12}$ lithiation method, ${ }^{13}$ use of chiral epoxides under phase transfer catalysts. ${ }^{14}$

However, very few methods are available for the synthesis of 1,4-benzoxazinone or 1,4pyridooxazinone derivatives. They are usually prepared by direct cyclization of 2-haloacetyl halide or alkyl 2-halo propionates with 2-aminophenol or 2-amino-3-hydroxypyridine. This is the most straightforward method by using 2-aminophenol or 2-nitrophenol as starting materials to 
obtain 1,4-benzoxazinones and a similar approach is also applied for pyridine series. Recently Chaudhuri and coworkers reported the palladium catalyzed C-C bond formation for the construction of different heterocyclic compounds. ${ }^{15}$ Very recently Dai and his group described one-pot synthesis of 1,4-benzoxazinones under microwave condition. ${ }^{16}$ However, many of these procedures have major drawbacks such as low yields of the products, long reaction time, harsh reaction conditions, difficulties in work-up, and the use of stoichiometric amounts and/or relatively expensive reagents. The majority of the existing methods are not useful to synthesize the different substituted benzoxazinones.

Our interest in development of new methodologies ${ }^{17}$ and experience in the synthesis of 1,4pyridooxazinones via Smiles rearrangement ${ }^{18,19}$ resulted in identifying a new method for the synthesis of 1,4-benzoxazinone derivatives. Herein we report a simple and efficient synthesis of 1,4-benzoxazinones by using substituted 2-chlorophenols as readily available starting material. To the best of our knowledge this is the first method for the synthesis of 1,4-benzoxazinone derivatives from 2-chlorophenols which is useful to prepare a large number of substituted 1,4benzoxazinones.

\section{Results and Discussion}

Initially, 2-chloro-4-methylphenol 1a was treated with $N$-benzyl-2-chloroacetamide in the presence of potassium carbonate in acetonitrile. The substituted acetamide $2 \mathbf{a}$ thus obtained was cyclized with cesium carbonate in DMF to give the corresponding 1,4-benzoxazinones 3a in good yield via Smiles type of rearrangement (Scheme 1). It is interesting to note that acetamide 2a undergoes Smiles type of rearrangement to form 7-methyl-2H-1,4-benzoxazin-3(4H)-one (3a) instead of the 6-methyl substituted product 3a'.

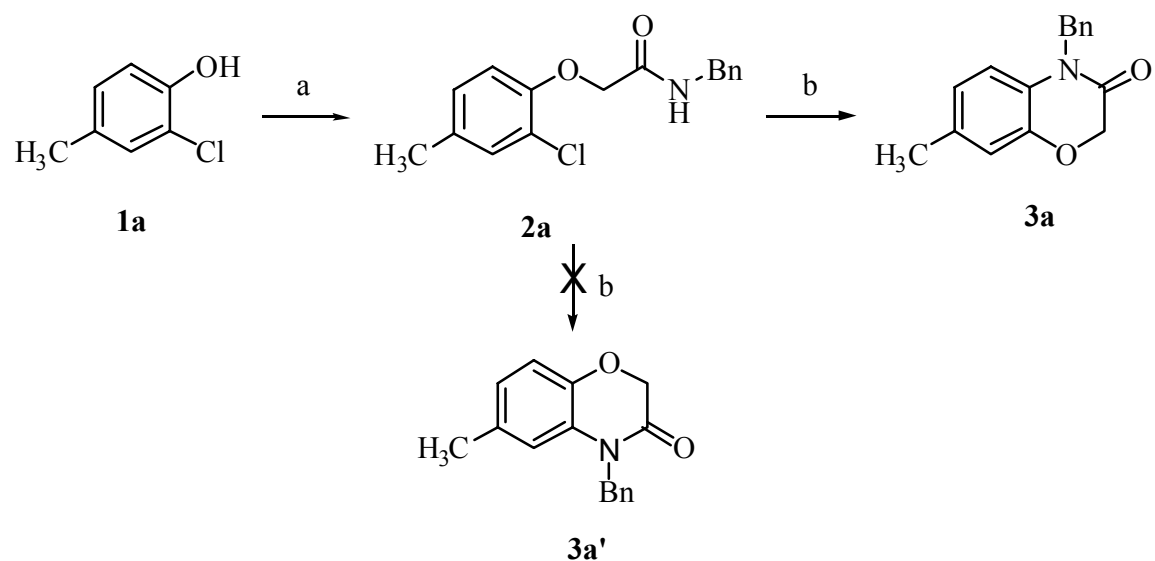

Scheme 1. Reagents and Conditions: a) $\mathrm{ClCH}_{2} \mathrm{CONHBn}, \mathrm{K}_{2} \mathrm{CO}_{3}, \mathrm{CH}_{3} \mathrm{CN}, \mathrm{rt}, 6 \mathrm{~h}, 95 \%$; b) $\mathrm{Cs}_{2} \mathrm{CO}_{3}$, DMF, reflux, $6 \mathrm{~h}, 68 \%$. 
The formation of 3a was confirmed by the reaction of 5-methyl-2-amino phenol (4) with 2chloroacetyl chloride using known methods (Scheme 2). Thus 2-chloroacetyl chloride was reacted with 4 to afford the intermediate 5 which was further cyclized (to give 6) followed by treatment with benzyl chloride furnished the compound 3a. The two compounds obtained by different synthetic routes were compared using physical and spectral data which was found to be same.

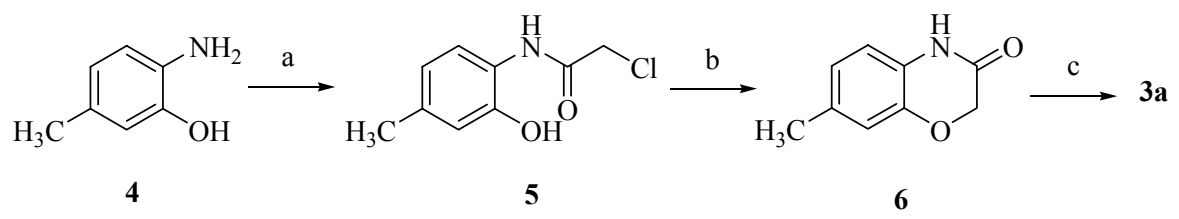

Scheme 2. Reagents and Conditions: (a) $\mathrm{ClCH}_{2} \mathrm{COCl}, \mathrm{K}_{2} \mathrm{CO}_{3}, \mathrm{CH}_{3} \mathrm{CN}$, rt; (b) $\mathrm{K}_{2} \mathrm{CO}_{3}$, DMF, reflux; (c) $\mathrm{BnCl}, \mathrm{K}_{2} \mathrm{CO}_{3}$, DMF, reflux.

The above results encouraged us to prepare various 1,4-benzoxazinone derivatives (3a-j) from different substituted 2-chloro phenols by the Smiles rearrangement. Accordingly 2-chloro phenols having methyl, methoxy, chloro and nitro substitution 1a-j (entries 1 to 10, Table 1), were treated with $\mathrm{N}$-benzyl-2-chloroacetamide in the presence of potassium carbonate in acetonitrile. The substituted acetamides $\mathbf{2 a - j}$ thus obtained were cyclized with cesium carbonate in DMF under refluxing conditions to give the corresponding 1,4-benzoxazinones 3a-j in good yields and the results are summarized in Table 1.

We also investigated the base and solvent effects on the synthesis of $\mathbf{3 a}$ and we found that $\mathrm{K}_{2} \mathrm{CO}_{3}$ in $\mathrm{CH}_{3} \mathrm{CN}$ was the most effective system ( $90 \%$ yield). Whereas $\mathrm{K}_{2} \mathrm{CO}_{3}$ in DMF, $\mathrm{Cs}_{2} \mathrm{CO}_{3}$ in $\mathrm{CH}_{3} \mathrm{CN}$ and in DMF were found to be less effective. On the other hand, $\mathrm{Cs}_{2} \mathrm{CO}_{3}$ in DMF was found to be more effective (66\% yield) for the cyclization of $\mathbf{2 a}$ when compared with other bases such as $\mathrm{Li}_{2} \mathrm{CO}_{3}, \mathrm{Na}_{2} \mathrm{CO}_{3}, \mathrm{~K}_{2} \mathrm{CO}_{3}$, and $\mathrm{Ag}_{2} \mathrm{CO}_{3}$. 
Table 1. Synthesis of benzo[b][1,4]oxazin-3-ones from 2-chlorophenols

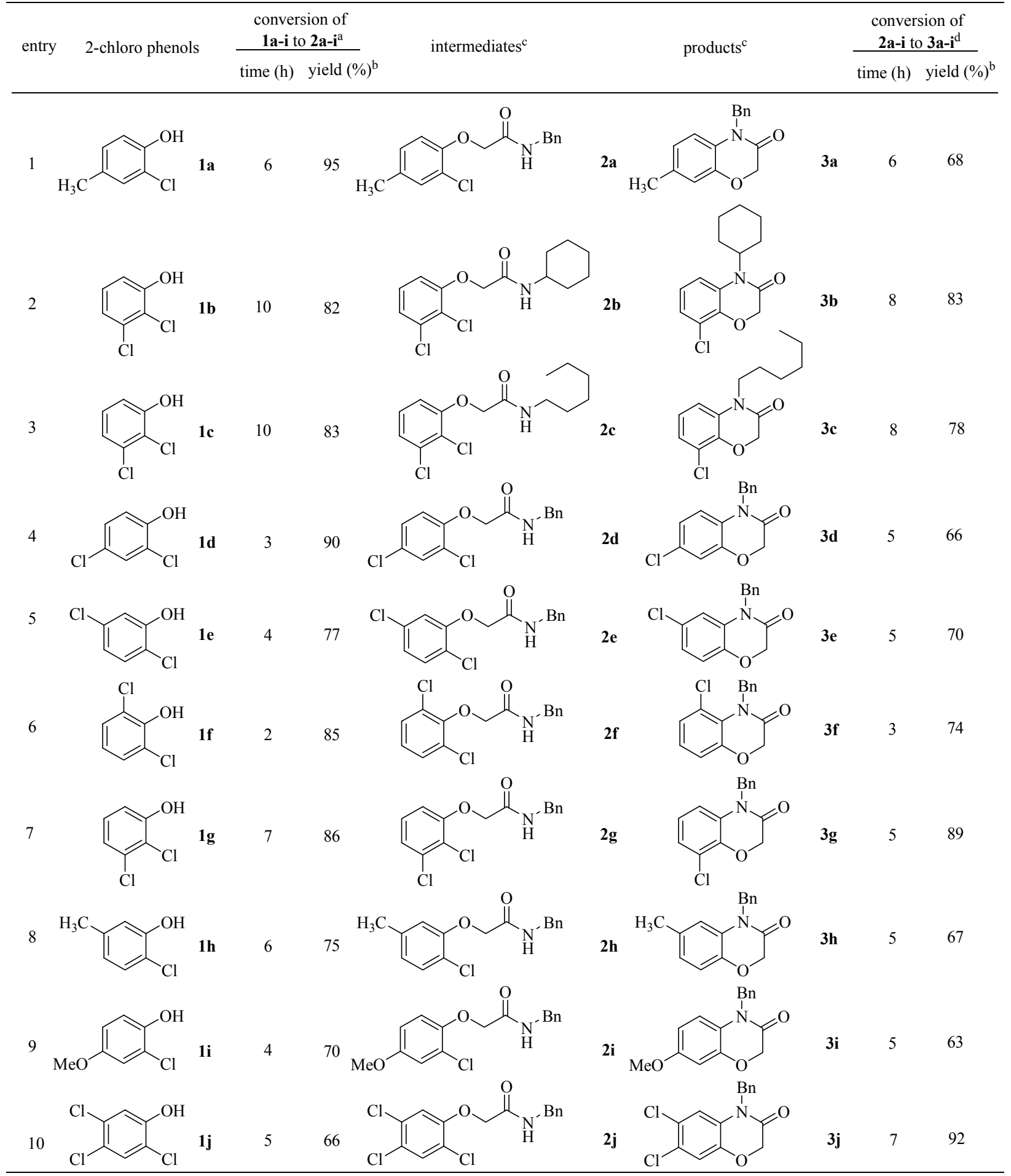

${ }^{a}$ Reaction conditions: $\mathrm{ClCH}_{2} \mathrm{CONHR}, \mathrm{K}_{2} \mathrm{CO}_{3}, \mathrm{CH}_{3} \mathrm{CN}$, rt. ${ }^{b}$ Isolated yields. ${ }^{\mathrm{c}} \mathrm{All}$ the products were characterized by ${ }^{1} \mathrm{H}$ and ${ }^{13} \mathrm{C}$ NMR and mass spectrometry. ${ }^{\mathrm{d}}$ Reaction conditions: $\mathrm{Cs}_{2} \mathrm{CO}_{3}$, DMF, reflux. 
The cyclization of $O$-alkylated product by Smiles rearrangement occurred via two steps: the spiro-type intermediate was formed in the first step, and was rearranged in the second step with the loss of $\mathrm{HCl}$ to afford compound 3a (Scheme 3).

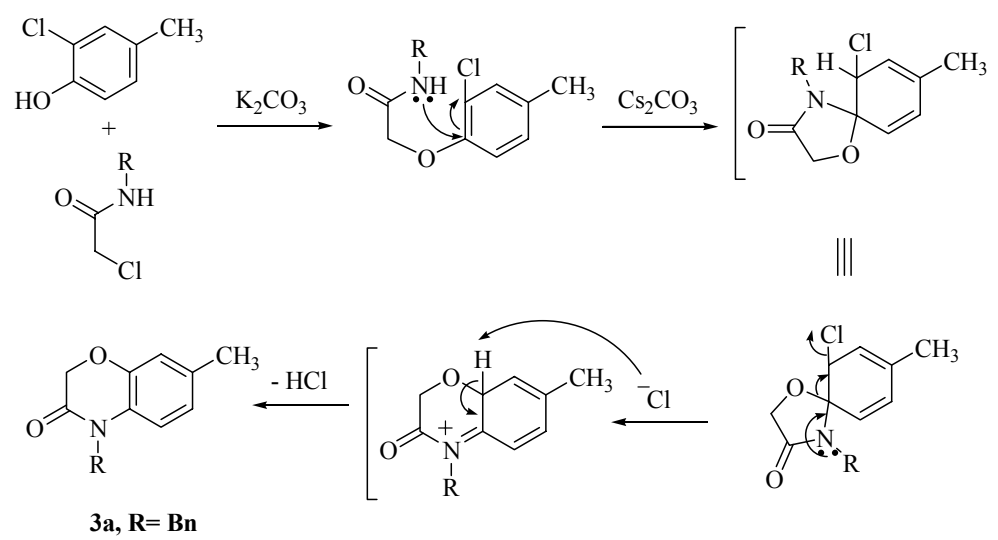

\section{Scheme 3}

\section{Conclusions}

In summary we have developed a novel and efficient method for the synthesis of a wide range of 1,4-benzoxazinone derivatives via Smiles rearrangement. Further work including the application, chemical transformation and biological activity is underway in our laboratory.

\section{Experimental Section}

General Procedures. ${ }^{1} \mathrm{H}$ and ${ }^{13} \mathrm{C}$ NMR spectra were recorded in $\mathrm{CDCl}_{3}$ (400 $\mathrm{MHz}$ for ${ }^{1} \mathrm{H}$ and $100 \mathrm{MHz}$ for ${ }^{13} \mathrm{C}$, respectively) with TMS as the internal reference on Bruker Advance $400 \mathrm{FT}$ spectrometer. Chemical shifts were reported in parts per million. Mass spectra (MS) were measured by the EI method. Melting points are uncorrected. Silica gel (70-230 mesh) was used for flash column chromatography. All the reactions were monitored by TLC using $0.25 \mathrm{~mm}$ silica gel plates (Merck 60F-254) with or without UV indicator. Acetonitrile was distilled from anhydrous calcium sulfate and $N, N$-dimethylformamide was distilled from anhydrous magnesium sulfate prior to use. All other reagents were commercially available (Acros, Aldrich) and were used without further purification. All microwave-assisted reactions were carried out on KMIC-1.5KW creator from Korea Microwave Instrument Company. The microwave-assisted reaction time is the hold time at the final temperature. 
General experimental procedure for $\boldsymbol{N}$-substituted-2-chloroacetamide for products 2 a to $2 \mathbf{j}$ The solution of 2-chlorophenol (1a to $\mathbf{1 j})$ (1 mmol), $N$-benzyl-2-chloroacetamide (1.1 mmol), $\mathrm{K}_{2} \mathrm{CO}_{3}(1.1 \mathrm{mmol})$ and $\mathrm{CH}_{3} \mathrm{CN}(20 \mathrm{ml})$ was refluxed for $3 \mathrm{~h}$. After completion of the reaction, the solution was cooled; solvent was evaporated under reduced pressure. The residue was poured into water and adjusted the $\mathrm{pH}$ 6-7 and extracted with ethyl acetate, washed with brine and dried over anhydrous $\mathrm{MgSO}_{4}$ to obtain the corresponding $N$-substituted-2-chloroacetamide (2a to $\mathbf{2 j}$ ).

$\boldsymbol{N}$-Benzyl-2-(2-chloro-4-methylphenoxy)acetamide (2a). Light yellow-brown crystal, mp 94-95 ${ }^{\circ} \mathrm{C} .{ }^{1} \mathrm{H}$ NMR $\left(400 \mathrm{MHz}, \mathrm{CDCl}_{3}\right) \delta 2.25(\mathrm{~s}, 3 \mathrm{H}), 4.52(\mathrm{~s}, 2 \mathrm{H}), 4.54(\mathrm{~d}, J=6.0 \mathrm{~Hz}, 2 \mathrm{H}), 6.77$ $(\mathrm{d}, J=8.4 \mathrm{~Hz}, 1 \mathrm{H}), 6.99(\mathrm{dd}, J=8.4,2.0 \mathrm{~Hz}, 1 \mathrm{H}), 7.16(\mathrm{~d}, J=2.0 \mathrm{~Hz}, 1 \mathrm{H}), 7.22(\mathrm{br}, 1 \mathrm{H})$, 7.26-7.35 (m, 5H); ${ }^{13} \mathrm{C}$ NMR (100 MHz, $\left.\mathrm{CDCl}_{3}\right) \delta 20.4,43.0,68.5,114.1,122.6,127.6,128.5$, 128.8, 130.9, 132.9, 137.8, 150.7, 167.8; MS (EI) m/z: $289\left(\mathrm{M}^{+}, 6 \%\right), 254$ (97), 148 (77), 125 (12), 107 (42), 91 (100), 77(24), 65 (12).

$\mathrm{N}$-Cyclohexyl-2-(2,3-dichlorophenoxy)acetamide (2b). White solid, mp 163-164 ${ }^{\circ} \mathrm{C} ;{ }^{1} \mathrm{H}$ NMR $\left(400 \mathrm{MHz}, \mathrm{CDCl}_{3}\right) \delta 1.23-1.31(\mathrm{~m}, 3 \mathrm{H}), 1.37-1.47(\mathrm{~m}, 2 \mathrm{H}), 1.60-1.64(\mathrm{~m}, 1 \mathrm{H}), 1.70-1.76(\mathrm{~m}$, 2H), 1.92-1.96 (m, 2H), 3.85-3.94 (m, 1H), 4.51 (s, 2H), $6.75(\mathrm{br}, 1 \mathrm{H}), 6.81(\mathrm{dd}, J=6.8,2.0 \mathrm{~Hz}$, 1H), 7.15-7.27 (m, 2H); ${ }^{13} \mathrm{C}$ NMR (100 MHz, $\left.\mathrm{CDCl}_{3}\right) \delta 24.6,25.5,32.9,47.8,68.3,111.7$,

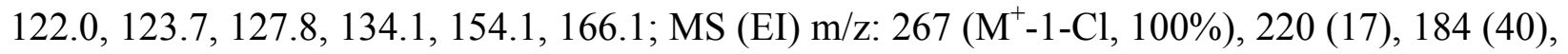
175 (15), 140 (15), 83 (26), 55 (17).

2-(2,3-Dichlorophenoxy)- $N$-hexylacetamide (2c). White solid, mp 96-98 ${ }^{\circ} \mathrm{C} ;{ }^{1} \mathrm{H}$ NMR $(400$ $\left.\mathrm{MHz}, \mathrm{CDCl}_{3}\right) \delta 0.89(\mathrm{t}, J=6.8 \mathrm{~Hz}, 3 \mathrm{H}), 1.26-1.39(\mathrm{~m}, 6 \mathrm{H}), 1.53-1.61(\mathrm{~m}, 2 \mathrm{H}), 3.37$ (dd, $J=13.2,6.8 \mathrm{~Hz}, 2 \mathrm{H}), 4.53(\mathrm{~s}, 2 \mathrm{H}), 6.82(\mathrm{dd}, J=7.6,2.0 \mathrm{~Hz}, 2 \mathrm{H}), 7.15-7.27(\mathrm{~m}, 2 \mathrm{H}) ;{ }^{13} \mathrm{C} \mathrm{NMR}$ $\left(100 \mathrm{MHz}, \mathrm{CDCl}_{3}\right) \delta 14.0,22.6,26.5,29.4,31.4,39.1,68.3,111.6,121.9,123.7,127.8,134.2$, 154.1, 167.0; MS (EI) m/z: 268 ( $\left.\mathrm{M}^{+}-1-\mathrm{Cl}, 100 \%\right), 184$ (10), 142 (9), 43 (12).

$\mathrm{N}$-Benzyl-2-(2,4-dichlorophenoxy)acetamide (2d). Colorless crystal, mp 124-125 ${ }^{\circ} \mathrm{C} ;{ }^{1} \mathrm{H}$ NMR $\left(400 \mathrm{MHz}, \mathrm{CDCl}_{3}\right) \delta 4.54(\mathrm{~s}, 2 \mathrm{H}), 4.55(\mathrm{~d}, J=6.0 \mathrm{~Hz}, 2 \mathrm{H}), 6.82(\mathrm{~d}, J=8.8 \mathrm{~Hz}, 1 \mathrm{H}), 7.11$ (br, $1 \mathrm{H}), 7.19(\mathrm{dd}, J=8.8,2.4 \mathrm{~Hz}, 1 \mathrm{H}), 7.26-7.37(\mathrm{~m}, 6 \mathrm{H}) ;{ }^{13} \mathrm{C} \mathrm{NMR}\left(100 \mathrm{MHz}, \mathrm{CDCl}_{3}\right) \delta 43.1$, $68.5,114.8,123.8,127.5,127.6,128.0,128.8,130.2,137.6,151.6,167.1 ; \mathrm{MS}(\mathrm{EI}) \mathrm{m} / \mathrm{z}: 309\left(\mathrm{M}^{+}\right.$, 4\%), 274 (32), 207 (19), 148 (100), 107 (33), 91 (96).

N-Benzyl-2-(2,5-dichlorophenoxy)acetamide (2e). White solid, mp 97-99 ${ }^{\circ} \mathrm{C} ;{ }^{1} \mathrm{H}$ NMR (400 $\left.\mathrm{MHz} \mathrm{CDCl}_{3}\right) \delta 4.54(\mathrm{~s}, 2 \mathrm{H}), 4.56(\mathrm{~d}, J=6 \mathrm{~Hz}, 2 \mathrm{H}), 6.89(\mathrm{~d}, J=2.0 \mathrm{~Hz}, 1 \mathrm{H}), 6.96(\mathrm{dd}, J=8.4$, $2.0 \mathrm{~Hz}, 1 \mathrm{H}), 7.12(\mathrm{br}, 1 \mathrm{H}), 7.26-7.36(\mathrm{~m}, 6 \mathrm{H}) ;{ }^{13} \mathrm{C} \mathrm{NMR}\left(100 \mathrm{MHz}, \mathrm{CDCl}_{3}\right) \delta 43.1,68.2,114.6$, 121.4, 123.0, 127.6, 127.7, 128.8, 131.0, 133.5, 137.6, 153.1, 167.0; MS (EI) m/z: 309 (M+, 5\%), 274 (57), 207 (15), 148 (92), 107 (35), 91 (100).

$\mathrm{N}$-Benzyl-2-(2,6-dichlorophenoxy)acetamide (2f). Colorless crystal, mp $94-96{ }^{\circ} \mathrm{C}$; ${ }^{1} \mathrm{H}$ NMR $\left(400 \mathrm{MHz}, \mathrm{CDCl}_{3}\right) \delta 4.58(\mathrm{~s}, 2 \mathrm{H}), 4.59(\mathrm{~d}, J=6.0 \mathrm{~Hz}, 2 \mathrm{H}), 7.03(\mathrm{t}, J=8.0 \mathrm{~Hz}, 1 \mathrm{H}), 7.26-7.38$ $(\mathrm{m}, 7 \mathrm{H}) ;{ }^{13} \mathrm{C} \mathrm{NMR}\left(100 \mathrm{MHz}, \mathrm{CDCl}_{3}\right) \delta 43.1,71.5,126.0,127.6,127.8,128.8,129.0,137.7$, 150.2, 167.6; MS (EI) m/z: 309 (M+2\%), 274 (16), 148 (100), 107 (41), 91 (80).

$\boldsymbol{N}$-Benzyl-2-(2,3-dichlorophenoxy)acetamide (2g). White solid, mp 129-130 ${ }^{\circ} \mathrm{C} ;{ }^{1} \mathrm{H}$ NMR (400 $\left.\mathrm{MHz} \mathrm{CDCl}_{3}\right) \delta 4.57(\mathrm{~d}, J=6.0 \mathrm{~Hz}, 2 \mathrm{H}), 4.59(\mathrm{~s}, 2 \mathrm{H}), 6.83(\mathrm{dd}, J=7.6,2.0 \mathrm{~Hz}, 1 \mathrm{H}), 7.13(\mathrm{br}$, 1H), 7.14-7.20 (m, 2H), 7.26-7.37 (m, 5H); $\left.{ }^{13} \mathrm{C} \mathrm{NMR} \mathrm{(100} \mathrm{MHz,} \mathrm{CDCl}_{3}\right) \delta 43.1,68.4,111.8$, 
122.1, 123.9, 127.6, 127.7, 128.8, 134.2, 137.6, 154.0, 167.1; MS (EI) m/z: $309\left(\mathrm{M}^{+}, 2 \%\right), 274$ (55), 148 (95), 107 (43), 91 (100), 77 (11), 65 (12).

N-Benzyl-2-(2-chloro-5-methylphenoxy)acetamide (2h). Light brown crystal, mp 113-114 ${ }^{\circ} \mathrm{C}$; ${ }^{1} \mathrm{H}$ NMR $\left(400 \mathrm{MHz}, \mathrm{CDCl}_{3}\right) \delta 2.32(\mathrm{~s}, 3 \mathrm{H}), 4.55(\mathrm{~s}, 2 \mathrm{H}), 4.56(\mathrm{~d}, J=6 \mathrm{~Hz}, 2 \mathrm{H}), 6.71(\mathrm{~s}, 1 \mathrm{H})$, $6.77(\mathrm{dd}, J=8,0.8 \mathrm{~Hz}, 1 \mathrm{H}), 7.20(\mathrm{br}, 1 \mathrm{H}), 7.22-7.36(\mathrm{~m}, 5 \mathrm{H}) ;{ }^{13} \mathrm{C} \mathrm{NMR}\left(100 \mathrm{MHz}, \mathrm{CDCl}_{3}\right) \delta$ 21.3, 43.0, 68.2, 114.9, 120.0, 123.6, 127.5, 127.6, 128.8, 130.0, 137.7, 138.5, 152.5, 167.7; MS (EI) m/z: 289 (M+, 3\%), 254 (100), 148 (58), 125 (12), 107 (36), 91 (91), 77 (21), 65 (12).

N-Benzyl-2-(2-chloro-4-methoxyphenoxy)acetamide (2i). White solid, mp 88-89 ${ }^{\circ} \mathrm{C} ;{ }^{1} \mathrm{H}$ NMR $\left(400 \mathrm{MHz}, \mathrm{CDCl}_{3}\right) \delta 3.75(\mathrm{~s}, 3 \mathrm{H}), 4.52(\mathrm{~s}, 2 \mathrm{H}), 4.55(\mathrm{~d}, J=6.0 \mathrm{~Hz}, 2 \mathrm{H}), 6.75(\mathrm{dd}, J=9.2,2.8$ $\mathrm{Hz}, 1 \mathrm{H}), 6.84(\mathrm{~d}, J=8.8 \mathrm{~Hz}, 1 \mathrm{H}), 6.93(\mathrm{~d}, J=2.8 \mathrm{~Hz}, 1 \mathrm{H}), 7.21(\mathrm{br}, 1 \mathrm{H}), 7.27-7.36(\mathrm{~m}, 5 \mathrm{H}) ;{ }^{13} \mathrm{C}$ NMR $\left(100 \mathrm{MHz}, \mathrm{CDCl}_{3}\right) \delta 43.0,55.9,69.2,113.2,115.7,116.1,123.8,127.6,128.8,137.8$, 147.1, 155.0, 167.9; MS (EI) m/z: 305 (M+27\%), 270 (56), 158 (30), 148 (63), 126 (10), 107 (35), 91 (100), 77 (16), 65 (16).

$\boldsymbol{N}$-Benzyl-2-(2,4,5-trichlorophenoxy)acetamide (2j). White crystal, mp 137-138 ${ }^{\circ} \mathrm{C}$; ${ }^{1} \mathrm{H}$ NMR $\left(400 \mathrm{MHz}, \mathrm{CDCl}_{3}\right) \delta 4.53(\mathrm{~s}, 2 \mathrm{H}), 4.55(\mathrm{~d}, J=6.0 \mathrm{~Hz}, 2 \mathrm{H}), 6.99(\mathrm{~s}, 1 \mathrm{H}), 7.06(\mathrm{br}, 1 \mathrm{H}), 7.27-7.36$ $(\mathrm{m}, 5 \mathrm{H}), 7.45(\mathrm{~s}, 1 \mathrm{H}) ;{ }^{13} \mathrm{C} \mathrm{NMR}\left(100 \mathrm{MHz}, \mathrm{CDCl}_{3}\right) \delta 43.2,68.5,115.6,122.1,126.0,127.6$, 127.8, 128.9, 131.1, 131.7, 137.5, 151.7, 166.6; MS (EI) m/z: 343 (M+, 4\%), 308 (22), 281 (11), 207 (26), 148 (100), 107 (34), 91 (95).

General experimental procedure for substituted $2 H$-1,4-benzoxazin-3(4H)-ones for products $3 \mathbf{a}$ to $3 \mathbf{j}$

The solution of $N$-substituted-2-(2-chlorophenoxy)acetamide (2a to $\mathbf{2} \mathbf{j}$ ) (1 mmol), $\mathrm{Cs}_{2} \mathrm{CO}_{3}$ (1.3 $\mathrm{mmol})$ in DMF $(20 \mathrm{~mL})$ was refluxed for $5 \mathrm{~h}$. After completion of the reaction the solvent was removed and the residue was poured into the water. The $\mathrm{pH}$ was adjusted to 6-7 and extracted with ethyl acetate. The ethyl acetate extract was washed with brine and dried over anhydrous $\mathrm{MgSO}_{4}$. The residue obtained was purified by silica gel column chromatography to obtain the corresponding substituted benzo[b][1,4] oxazin-3(4H)-ones (3a to $\mathbf{3 j}$ ).

4-Benzyl-7-methyl-2H-1,4-benzoxazin-3(4H)-one (3a). White solid; mp 90-91 ${ }^{\circ} \mathrm{C}$; ${ }^{1} \mathrm{H}$ NMR $\left(400 \mathrm{MHz}, \mathrm{CDCl}_{3}\right) \delta 2.24(\mathrm{~s}, 3 \mathrm{H}), 4.69(\mathrm{~s}, 2 \mathrm{H}), 5.13(\mathrm{~s}, 2 \mathrm{H}), 6.67-6.80(\mathrm{~m}, 3 \mathrm{H}), 7.23-7.32(\mathrm{~m}$, $5 \mathrm{H}) ;{ }^{13} \mathrm{C} \mathrm{NMR}\left(100 \mathrm{MHz}, \mathrm{CDCl}_{3}\right) \delta 20.7,45.0,67.8,115.5,117.6,123.3,126.2,126.6,127.5$, 128.9, 134.1, 136.1, 145.1, 164.6; MS (EI) m/z: 253 (M+, 96\%), 134 (8), 91 (100), 65 (9).

8-Chloro-4-cyclohexyl-2H-1,4-benzoxazin-3(4H)-one (3b). Light pink solid, mp 74-76 ${ }^{\circ} \mathrm{C} ;{ }^{1} \mathrm{H}$ NMR $\left(400 \mathrm{MHz}, \mathrm{CDCl}_{3}\right) \delta 1.22-1.43(\mathrm{~m}, 3 \mathrm{H}), 1.71(\mathrm{t}, 1 \mathrm{H}), 1.79-1.91(\mathrm{~m}, 4 \mathrm{H}), 2.30-2.40(\mathrm{~m}$, $2 \mathrm{H}), 4.10-4.18(\mathrm{~m}, 1 \mathrm{H}), 4.56(\mathrm{~s}, 2 \mathrm{H}), 6.95(\mathrm{t}, J=8.0 \mathrm{~Hz}, 1 \mathrm{H}), 7.06-7.09(\mathrm{~m}, 2 \mathrm{H}) ;{ }^{13} \mathrm{C} \mathrm{NMR}(100$ $\left.\mathrm{MHz}_{\mathrm{CDCl}}\right) \delta 25.3,26.4,29.4,57.5,68.9,114.8,122.6,122.7,124.7,131.3,142.8,165.7$; MS (EI) m/z: $265\left(\mathrm{M}^{+}, 23 \%\right), 183$ (100), 154 (45), 55 (14), 44 (10).

8-Chloro-4-hexyl-2H-1,4-benzoxazin-3(4H)-one (3c). Colorless liquid; ${ }^{1} \mathrm{H}$ NMR (400 MHz, $\left.\mathrm{CDCl}_{3}\right) \delta 0.89(\mathrm{t}, J=6.8 \mathrm{~Hz}, 3 \mathrm{H}), 1.31-1.39(\mathrm{~m}, 6 \mathrm{H}), 1.60-1.68(\mathrm{~m}, 2 \mathrm{H}), 3.91(\mathrm{t}, J=7.6 \mathrm{~Hz}, 2 \mathrm{H})$, $4.67(\mathrm{~s}, 2 \mathrm{H}), 6.89(\mathrm{dd}, J=8.0,1.6 \mathrm{~Hz}, 1 \mathrm{H}), 6.96(\mathrm{t}, J=8.0 \mathrm{~Hz}, 1 \mathrm{H}), 7.05(\mathrm{dd}, J=8.0,1.6 \mathrm{~Hz})$; ${ }^{13} \mathrm{C}$ NMR $\left(100 \mathrm{MHz}, \mathrm{CDCl}_{3}\right) \delta 14.0,22.6,26.5,27.0,41.5,67.7,113.4,122.5,122.7,124.5$, 
130.0, 141.5, 163.6; MS (EI) m/z: 267 (M+, 51\%), 236 (19), 225 (12), 207 (11), 196 (19), 183 (100), 168 (65), 154 (43), 141 (15), 99 (11), 77(10), 41 (12).

4-Benzyl-7-chloro-2H-1,4-benzoxazin-3(4H)-one (3d). Off-white solid, mp 98-100 ${ }^{\circ} \mathrm{C}$; ${ }^{1} \mathrm{H}$ NMR (400 MHz, CDCl3) $\delta 4.72(\mathrm{~s}, 2 \mathrm{H}), 5.13(\mathrm{~s}, 2 \mathrm{H}), 6.77(\mathrm{~d}, J=8.4 \mathrm{~Hz}, 1 \mathrm{H}), 6.85$ (dd, $J=8.8$, $2.4 \mathrm{~Hz}, 1 \mathrm{H}), 6.99(\mathrm{~d}, \mathrm{~J}=2.4 \mathrm{~Hz}, 1 \mathrm{H}), 7.21-7.33(\mathrm{~m}, 5 \mathrm{H}) ;{ }^{13} \mathrm{C} \mathrm{NMR}\left(100 \mathrm{MHz}, \mathrm{CDCl}_{3}\right) \delta 45.0$, 67.6, 116.5, 117.4, 122.7, 126.6, 127.7, 128.9, 129.0, 135.5, 145.9, 164.1; MS (EI) m/z: $273\left(\mathrm{M}^{+}\right.$, 44\%), 207 (17), 91 (100).

4-Benzyl-6-chloro-2H-1,4-benzoxazin-3(4H)-one (3e). White solid, mp 113-114 ${ }^{\circ} \mathrm{C} ;{ }^{1} \mathrm{H} \mathrm{NMR}$ $\left(400 \mathrm{MHz}, \mathrm{CDCl}_{3}\right) \delta 4.71(\mathrm{~s}, 2 \mathrm{H}), 5.11(\mathrm{~s}, 2 \mathrm{H}), 6.87-6.91(\mathrm{~m}, 3 \mathrm{H}), 7.23-7.35(\mathrm{~m}, 5 \mathrm{H}) ;{ }^{13} \mathrm{C} \mathrm{NMR}$ $\left(100 \mathrm{MHz}, \mathrm{CDCl}_{3}\right) \delta 45.0,76.8,115.8,118.0,123.7,126.7,127.8,129.0,129.8,135.3,143.9$, 164.4; MS (EI) m/z: 273 (M+, 43\%), 253 (5), 207 (29), 91 (100), 65 (7), 44 (12).

4-Benzyl-5-chloro-2H-1,4-benzoxazin-3(4H)-one (3f). Colorless liquid; ${ }^{1} \mathrm{H}$ NMR (400 MHz, $\left.\mathrm{CDCl}_{3}\right) \delta 4.54(\mathrm{~s}, 2 \mathrm{H}), 5.51(\mathrm{~s}, 2 \mathrm{H}), 6.87-6.98(\mathrm{~m}, 3 \mathrm{H}), 7.13-7.23(\mathrm{~m}, 5 \mathrm{H}) ;{ }^{13} \mathrm{C} \mathrm{NMR}(100 \mathrm{MHz}$, $\left.\mathrm{CDCl}_{3}\right) \delta 47.0,69.2,115.8,123.9,125.5,126.0,127.0,127.7,128.4,136.4,150.9,167.3$; MS (EI) m/z: $273\left(\mathrm{M}^{+}, 39 \%\right), 207$ (8), 91 (100), 65 (8).

4-Benzyl-8-chloro-2H-1,4-benzoxazin-3(4H)-one (3g). White solid, mp $98-99{ }^{\circ} \mathrm{C}$; ${ }^{1} \mathrm{H}$ NMR $\left(400 \mathrm{MHz}, \mathrm{CDCl}_{3}\right) \delta 4.80(\mathrm{~s}, 2 \mathrm{H}), 5.14(\mathrm{~s}, 2 \mathrm{H}), 6.76-6.83(\mathrm{~m}, 2 \mathrm{H}), 7.02(\mathrm{dd}, J=7.6,2.0 \mathrm{~Hz}, 1 \mathrm{H})$, 7.21-7.33 (m, 5H); ${ }^{13} \mathrm{C}$ NMR (100 MHz, $\left.\mathrm{CDCl}_{3}\right) \delta 45.2,67.8,114.2,122.4,122.8,124.9,126.6$, 127.7, 129.0, 130.0, 135.5, 141.5, 164.2; MS (EI) m/z: $273\left(\mathrm{M}^{+}, 67 \%\right), 91$ (100).

4-Benzyl-6-methyl-2H-1,4-benzoxazin-3(4H)-one (3h). White solid; mp 117-119 ${ }^{\circ} \mathrm{C} ;{ }^{1} \mathrm{H}$ NMR $\left(400 \mathrm{MHz}, \mathrm{CDCl}_{3}\right) \delta 2.20(\mathrm{~s}, 3 \mathrm{H}), 4.68(\mathrm{~s}, 2 \mathrm{H}), 5.14(\mathrm{~s}, 2 \mathrm{H}), 6.69(\mathrm{~s}, 1 \mathrm{H}), 6.75(\mathrm{~d}, J=8.4 \mathrm{~Hz}$, $1 \mathrm{H}), 6.88(\mathrm{~d}, J=8.4 \mathrm{~Hz}, 1 \mathrm{H}), 7.24-7.34(\mathrm{~m}, 5 \mathrm{H}) ;{ }^{13} \mathrm{C} \mathrm{NMR}\left(100 \mathrm{MHz}, \mathrm{CDCl}_{3}\right) \delta$ 45.0, 55.6, $67.8,103.2,107.9,116.3,122.2,126.6,127.5,128.9,136.0,146.3,156.4,164.1 ; \mathrm{MS}(\mathrm{EI}) \mathrm{m} / \mathrm{z}$ : $253\left(\mathrm{M}^{+}, 63 \%\right), 134$ (11), 91 (100), 77 (11), 65 (16).

4-Benzyl-7-methoxy-2H-1,4-benzoxazin-3(4H)-one (3i). Light pink solid; mp $71-74{ }^{\circ} \mathrm{C} ;{ }^{1} \mathrm{H}$ NMR (400 MHz, $\left.\mathrm{CDCl}_{3}\right) \delta 3.71(\mathrm{~s}, 3 \mathrm{H}), 4.69(\mathrm{~s}, 2 \mathrm{H}), 5.12(\mathrm{~s}, 2 \mathrm{H}), 6.43(\mathrm{dd}, J=8.8,2.8 \mathrm{~Hz}$, $1 \mathrm{H}), 6.56(\mathrm{~d}, J=2.8 \mathrm{~Hz}, 1 \mathrm{H}), 6.76(\mathrm{~d}, J=12.8 \mathrm{~Hz}, 1 \mathrm{H}), 7.23-7.32(\mathrm{~m}, 5 \mathrm{H}) ;{ }^{13} \mathrm{C} \mathrm{NMR}(100 \mathrm{MHz}$, $\left.\mathrm{CDCl}_{3}\right) \delta 45.0,55.6,67.8,103.2,107.9,116.3,122.2,126.6,127.5,128.9,136.1,146.3,156.4$, 164.1; MS (EI) m/z: 269 (M+, 100\%), 178 (42), 150 (52), 135 (6), 120 (8), 91 (79), 65 (14).

4-Benzyl-6,7-dichloro-2H-1,4-benzoxazin-3(4H)-one (3j). White solid; mp $157-159{ }^{\circ} \mathrm{C} ;{ }^{1} \mathrm{H}$ NMR (400 MHz, $\left.\mathrm{CDCl}_{3}\right) \delta 4.72(\mathrm{~s}, 2 \mathrm{H}), 5.10(\mathrm{~s}, 2 \mathrm{H}), 6.94(\mathrm{~s}, 1 \mathrm{H}), 7.07(\mathrm{~s}, 1 \mathrm{H}), 7.22-7.36(\mathrm{~m}$, $5 \mathrm{H}) ;{ }^{13} \mathrm{C}$ NMR $\left(100 \mathrm{MHz}, \mathrm{CDCl}_{3}\right) \delta 45.1,67.6,116.9,118.6,126.1,126.7,126.9,128.0,128.5$, 129.1, 135.0, 144.3, 163.9; MS (EI) m/z: 307 (M+, 42\%), 91 (100), 65 (12).

\section{Acknowledgements}

The work is supported by Korean Research Foundation Grant (KRF-2005-210-C00025). 


\section{References and Notes}

1. (a) Wolfer, J.; Bekele, T.; Abraham, C. J.; Dogo-Isonagie, C.; Lectka, T. Angew. Chem. Int. Ed. 2006, 45, 7398. (b) Ilas, J.; Anderluh, P. S.; Dolenc, M. S.; Kikelji, D. Tetrahedron 2005, 61, 7325. (c) Zhou, Y.-G.; Yang, P.-Y.; Han, X.-W. J. Org. Chem. 2005, 70, 1679. (d) Matzanke, N.; Löwe, W.; Perachon, S.; Sokoloff, P.; Schwartz, J.; Stark, H. Eur. J. Med. Chem. 1999, 34, 791. (e) Vliet, L. A; Rodenhuis, N.; Dijkstra, D.; Wikström, H.; Thomas, A.; Kevin A.P.; Leonard T. S.; Thomas, G. M.; Lawrence, H. D.; Mary, E. W.; Rita, M. L; Svensson, H. K.; Sundell, S.; Lundmark, M. J. Med. Chem. 2000, 43, 2871.

2. Yamamoto, T.; Hor, M.; Watanabe, I.; Tsutsui, H. Chem. Pharm. Bull. 1999, 47, 22.

3. Chiu, H-I.; Lin, Y-C.; Cheng, C-Y.; Tsai, M-C.; Yu, H-C. Bioorg. Med. Chem. 2001, 9, 383.

4. Savelon, L.; Bizot-Espiard, J. G.; Caignard, D. H.; Pfeiffer, B.; Renard, P.; Viaud, M. C.; Guillaumet, G. Bioorg. Med. Chem. 1998, 6, 133.

5. Greenwood, D. T.; Mallion, K. B.; Todd, A. H.; Turner, R.W. J. Med. Chem. 1975, 18, 573.

6. Macchiarulo, A.; Costantino, G.; Fringuelli, D.; Vecchiarelli, A.; Schiaffella F.; Fringuelli, R. Bioorg. Med. Chem. 2002, 10, 3415.

7. (a) Marchi, M. B.; Claudia, N. A. S.; Mar tin, R. Langmuir 1977, 13, 3665. (b) Favaro, G.; Malatesta, V.; Mazzucato, U.; Millani, C.; Ottavi, G. Proceeding of the Indian Acad. of Sciences 1995, 107, 659. (c) Zhang, Y.; Hartman, S. R.; Moshary, F. J. Chem. Physics 1996, 104, 4371. (d) Guibin, M.; Soutang, J.; Wang, G.; Sun, Y. Spectroscopy and Spectral Anal. 1994, 14, 15.

8. (a) Liu, D.; Kamat, V. R. J. Electrochem. Soc. 1995, 142, 835. (b) Rose, D.; Lieske, E. Ger. Offen. DE 4, 1993, 122, 035; Chem. Abstr., 1993, 118, 154134p.

9. Afarinkia, K.; Bahar, A.; Neuss, J. Synlett 2003, 2341.

10. Afarinkia, K.; Bahar, A; Neus J.; Ruggiero, A. Tetrahedron Lett. 2004, 45, 3995.

11. McKillop, A.; Sayer, T. S. B. J. Org. Chem. 1976, 41, 1079.

12. (a) Bartsch, H.; Schwarz, O. J. Heterocycl. Chem. 1982, 1189. (b) Bartsch, H.; Ofner, M.; Schwarz, O.; Thomann, W. Heterocycles 1984, 22, 2789.

13. Chacun-Lefevre, L.; Buon, C.; Bouyssou, P.; Coudert, G. Tetrahedron Lett. 1998, 39, 5763.

14. Albanese, D.; Landini, D.; Penso, M. Chem. Commun. 1999, 2095.

15. Chaudhuri, G.; Chowdhury, C.; Kundu, N. G. Synlett 1998, 1273.

16. Feng G.-F.; Wu J.-L.; Dai W.-M. Tetrahedron 2006, 62, 4635.

17. (a) Ma, C.; Cho, S-D.; Falck, J. R.; Shin, D-S. Heterocycles 2004, 63, 75. (b) Cho, S-D.; Song, S-Y.; Park, Y-D.; Kim, J-J.; Joo, W-H.; Shiro, M.; Falck, J. R.; Shin D-S.; Yoon, Y-J. Tetrahedron Lett. 2003, 44, 8995. (c) Cho, S-D.; Park, Y-D.; Kim, J-J.; Joo, W-H.; Shiro, M.; Esser, L.; Falck, J. R.; Ahn, C.; Yoon, Y-J.; Shin, D-S. Tetrahedron 2004, 60, 3763.

18. (a) Cho, S.-D.; Park, Y.-D.; Kim, J-J.; Lee, S.-G.; Ma, C.; Song, S-Y.; Joo, W.-H.; Falck, J. R.; Shiro, M.; Yoon, Y.-J.; Shin, D.-S. J. Org. Chem. 2003, 68, 7918. (b) Shin, D.-S.; Park, J. K. Bull. Korean Chem. Soc. 2007, 2219. 
19. (a) Baker, W. R. J. Org. Chem. 1983, 48, 5140. (b) Coutts, I. G. C.; Southcott, M. R. J. Chem. Soc., Perkin Trans. 1 1990, 767. (c) Anelli, P. L.; Brocchetta, M.; Calabi, L.; Secchi, C.; Uggeri, F.; Verona, S. Tetrahedron 1997, 53, 11919. (d) Buchstaller, H.-P.; Anlauf, U. Synthesis 2005, 639. 